\title{
Síntomas Músculo-Esqueléticos en Trabajadores Operativos del Área de Mantenimiento de una Empresa Petrolera Ecuatoriana
}

\section{MUSCULOSKELETAL SYMPTOMS IN THE AREA OF OPERATIONAL MAINTENANCE OF AN OIL COMPANY WORKERS}

\author{
Agila-Palacios Enmanuel' ${ }^{1}$ Colunga-Rodríguez Cecilia ${ }^{2}$, González-Muñoz Elvia ${ }^{3}$, Delgado-García Diemen ${ }^{4}$ \\ 1. Médico de Salud Ocupacional de Inscora S.A. Doctorante de Ciencias de la Salud en el Trabajo, por la Universidad de Guadalajara, México. \\ 2. Instituto de Investigación en Salud Ocupacional (IISO), Universidad de Guadalajara; UMAE Hospital de Pediatría Centro Médico Nacional de Occidente, \\ Instituto Mexicano del Seguro Social. \\ 3. Centro de Investigaciones en Ergonomía, Centro Universitario de Arte, Arquitectura y Diseño de la Universidad de Guadalajara, México. \\ 4. Clínica Río Blanco. Docente de la Universidad Científica del Sur de Perú.
}

\begin{abstract}
RESUMEN
Contexto: Al ser considerada la ergonomía como una disciplina de carácter científico, que evalúa los riesgos ergonómicos en el medio ambiente laboral, puede evitar enfermedades ocupacionales y accidentes del trabajo, contribuyendo a mejorar las condiciones laborales en una organización. Objetivo: Determinar la prevalencia de síntomas músculo-esqueléticos en trabajadores operativos del puesto de trabajo mantenimiento de una Empresa Petrolera Ecuatoriana. Metodología: Se llevó a cabo un estudio transversal en 102 trabajadores de sexo masculino, correspondientes al puesto de trabajo de mantenimiento de una empresa petrolera, situada en una locación de la Provincia de Sucumbíos, durante el año 2013; la edad comprendida estuvo entre 18 y 49 años. Para la recolección de datos a cada uno de los trabajadores, previo consentimiento informado se aplicó: un Cuestionario Socio-Demográfico, una Historia Médica Ocupacional y el Cuestionario Nórdico Estandarizado. Resultados: La mayor prevalencia de síntomas músculo-esqueléticos, se encuentra en el grupo de trabajadores de entre 30 y 40 años de edad, en las regiones anatómicas: espalda baja 66 (64,7\%), seguido de espalda alta $44(43,1 \%)$, cuello $38(37,3 \%)$ y hombro 27 $(26,5 \%)$, siendo los más afectados los puestos de trabajo técnicoeléctrico y técnico-mecánico. La regresión logística binaria determinó que las variables no son estadísticamente significativas y, por lo tanto, por sí solas no explican la aparición de síntomas MúsculoEsqueléticos. Conclusión: Se concluye que existe una elevada prevalencia de síntomas músculo-esqueléticos en la población estudiada, por lo que se recomienda efectuar una evaluación ergonómica exhaustiva de los puestos de trabajo y posteriormente buscar mecanismos y estrategias de control y prevención de riesgos ergonómicos, con la finalidad de minimizar el desarrollo de lesiones músculo-esqueléticos en la población de estudio.
\end{abstract}

(Agila-Palacios E, Colunga-Rodríguez C, González-Muñoz E, Delgado-García D, 2014. Síntomas Músculo-Esqueléticos en Trabajadores Operativos del Área de Mantenimiento de una Empresa Petrolera Ecuatoriana. Cienc Trab. Sep-Dic; 16 [51]: 198-205).

Palabras clave: ERGONOMÍA, SÍNTOMAS MÚSCULO-ESQUELÉTICOS, FACTORES DE RIESGO, SALUD OCUPACIONAL, INDUSTRIA DEL PETRÓLEO Y GAS.

\section{ABSTRACT}

Context: When considered ergonomics as a scientific discipline that evaluates ergonomic hazards in the working environment, you can prevent occupational diseases and industrial accidents, helping to improve working conditions in an organization. Objective: To determine the prevalence of musculoskeletal symptoms in workers operating as a maintenance work Ecuadorian Oil Company. Methodology: A cross-sectional study was conducted on 102 male workers, job for the maintenance of an oil company, situated in a location in the province of Sucumbios, in 2013, the age range was between 18 and 49. To collect data for each worker, informed consent was applied: A Socio-Demographic Questionnaire an Occupational Medical History and the Standardized Nordic Questionnaire. Results: The highest prevalence of musculoskeletal symptoms are in the group of workers between 30 and 40 years old, in the anatomical regions: lower back $66(64,7 \%)$, followed by upper back $44(43,1 \%)$, neck $38(37,3 \%)$ and shoulder $27(26,5 \%)$ being the most affected stations mechanical technician and electrician work. Binary logistic regression determined that the variables are not statistically significant, and thus alone do not explain the occurrence of musculoskeletal symptoms. Conclusion: We conclude that there is a high prevalence of musculoskeletal symptoms in the study population, so it is recommended that a comprehensive ergonomic evaluation of jobs and then find mechanisms and strategies for control and prevention of ergonomic hazards, with the aim minimize the development of musculoskeletal injuries in the study population.

Key words: ERGONOMICS, MUSCULOSKELETAL SYMPTOMS, RISKS FACTORS, OCCUPATIONAL HEALTH, OIL AND GAS INDUSTRY.

\section{INTRODUCCIÓN}

La ergonomía, al ser considerada como una disciplina científica que aporta elementos para evaluar los riesgos ergonómicos producidos en el trabajo, previene enfermedades profesionales y lesiones en el trabajo, lo que facilita una mayor integración del

personal, mejorando las condiciones de trabajo y las relaciones

Diemen Delgado

Av. Santa Maria 777, Los Andes, Chile

Tel.: (34) 2590500

e-mail:diemen.delgado@clinicarioblanco.cl

Recibido: 28 de Mayo 2014 / Aceptado: 13 Septiembre 2014 
laborales del trabajador. Por lo tanto, uno de los objetivos de la ergonomía es la adaptación del trabajo al hombre en relación a sus requerimientos físicos en su medio de trabajo, sean estos sobreesfuerzos, posturas forzadas, movimientos repetitivos, 0 estrés de esfuerzo, Cuando el trabajador siente la incapacidad física para tolerar esta carga de trabajo se originan las lesiones músculo esqueléticas.

Según Kumar ${ }^{1}$ las lesiones músculo-esqueléticas son de naturaleza biomecánica, interviniendo a su vez 4 teorías: interacción multifactorial, fatiga diferencial, carga acumulada y esfuerzo excesivo, las cuales explican el origen de la lesión en primera instancia, en la ruptura traumática de los tejidos, con signos de inflamación; el proceso traumático en este momento sufre una alteración de la integridad de los tejidos y del orden mecánico, que sumado a la alteración de las propiedades visco-elástica de los tejidos, desencadena la lesión.

Merlino, Rosecranse, Antón y Cook definen las lesiones músculoesqueléticas relacionadas con el trabajo "como aquellas causadas por actividades netamente laborales, las cuales pueden ocasionar intensa sintomatología que puede agravarse, como dolor, parestesia, entumecimiento, en una o varias partes del cuerpo, incapacidad temporal o permanente, días de trabajo perdidos, incremento en los costos de compensación y disminución en el rendimiento del trabajo". ${ }^{2}$

Verdú, Villanueva ${ }^{3}$ indican que uno de los daños directos a la salud del trabajador, originados de la carga física de trabajo son precisamente las lesiones músculo-esqueléticas, apareciendo bien por la acumulación de pequeños traumatismos que se presentan paulatinamente en la actividad laboral o bien de manera inmediata, produciendo enfermedades profesionales músculo-esqueléticas y accidentes de trabajo por esfuerzos físicos.

Similarmente la Organización Internacional del Trabajo definió a las enfermedades del sistema músculo-esquelético, "como aquellas causadas por actividades inherentes al trabajo o por los factores de riesgo presentes en el ambiente laboral, posturas incorrectas o sin neutralidad, vibraciones y presencia de frío en el ambiente de trabajo, esfuerzos excesivos y concentración de fuerzas mecánicas y movimientos repetitivos. ${ }^{4}$

Entre las consecuencias ocupacionales producidas por las lesiones músculo-esqueléticas en los trabajadores, se distinguen el ausentismo, la disminución productiva, la modificación de la calidad de vida del trabajador, las incapacidades temporales o permanentes, los cambios en las perspectivas, el incremento de los costos económicos, actitudes psicosociales individuales y familiares, especialmente cuando las condiciones en el ambiente de trabajo no son una garantía de comodidad, productividad, seguridad y salud. ${ }^{4}$

Pruss, Corvalán, Pastides y Hollander ${ }^{5}$ indican que los trastornos músculo-esqueléticas en los Estados Unidos constituyen la primera causa de discapacidad, y en los servicios médicos de las empresas engloban más de 131 millones de visitas de pacientes al año.

Las estadísticas laborales del Ministerio de Trabajo y Asuntos Sociales de España ${ }^{6}$ informaron que los países que conforman la Unión Europea durante ese año reportaron 22.844 enfermedades laborales, de las cuales el 81,6 \% fue debido a lesiones músculoesqueléticas. De igual manera el Instituto de Seguridad e Higiene en el Trabajo de España durante el año 2003 entre los meses enero a junio, registró 1284 enfermedades de origen profesional y de ellas el 90,5\% corresponde a lesiones músculo-esqueléticas, de ahí el impacto que tienen estas lesiones en la salud física y mental de los trabajadores.

Troconis, Palma, Montiel, Quevedo, Rojas, Chacin, et al. ${ }^{7}$ señalan estadísticas sobre las enfermedades ocupacionales del Instituto de Salud y Seguridad Laboral de los trabajadores (INPSASEL) de Venezuela, entre ellas las de lesiones músculo-esqueléticas, que en el año 2006 se presentaron en un 76,5 \%, porcentaje muy alto de esta patología, que impide el normal desempeño de las actividades ocupacionales del trabajador.

En Venezuela, uno de los principales países productores de petróleo en el mundo, la industria petrolera reúne a un elevado número de trabajadores que laboran en operaciones de perforación, que por factores de riesgo especialmente ergonómicos físicos, exponen al trabajador a contraer lesiones músculo-esqueléticas. En Venezuela estas lesiones se ubican entre las 10 primeras enfermedades ocupacionales que afectan a los trabajadores, reflejando el grave problema existente en esta población laboral, lo cual indica la necesidad de implementar un programa de vigilancia médica de tipo osteo-muscular, para controlar, y evitar las lesiones músculo-esqueléticas. ${ }^{7}$

Localmente las cifras que maneja el Seguro de Riesgos del Trabajo del IESS, suman 2 mil muertes por año en Ecuador y 2,2 millones en el mundo, de los cuales el $86 \%$ se producen por enfermedades profesionales, según el último informe de la Organización Internacional del Trabajo. ${ }^{8}$

El Seguro de Riesgos del Trabajo (SRT) de Ecuador cubre dos contingencias básicas: accidentes de trabajo y enfermedades profesionales. El primero se enmarca en sucesos súbitos que afectan al trabajador en el desempeño de sus funciones causando incapacidad temporal o definitiva o incluso la muerte. El segundo, cuando inhabilitan al trabajador por factores de riesgo (enfermedades profesionales).

Debido al sub-registro con que cuenta el IESS en el Ecuador, ocurren 80 mil accidentes de trabajo al año y 60 mil enfermedades profesionales como hipoacusia, pérdida de capacidad visual, del olfato, trastornos músculo-esqueléticos y enfermedades por factores de riesgo psicosociales La organización precisa que en los países desarrollados se pierde el 4\% del Producto Interno Bruto (PIB) y en los países en vías de desarrollo, se habla de pérdidas entre el 8 al 9\% del PIB.

Las lesiones músculo-esqueléticas, de acuerdo a estadísticas proporcionadas, actualmente constituyen la principal fuente de ausentismo laboral; de los pocos datos extraídos en la entrevista de Riesgos del Trabajo del Ecuador (2013) el lumbago ocupó el $36 \%$ y, el síndrome de túnel del carpo $40 \%{ }^{8}$

La normativa nacional en Ecuador es mínima. De acuerdo con el Art. 9 del Reglamento del Sistema de Auditoria de Riesgos del Trabajo (R0 319: 19-diciembre-2011), en el numeral 2.1, literal a) expresa que "Se han identificado las categorias de factores de riesgo ocupacional de todos los puestos de trabajo, utilizando procedimientos reconocidos en el ámbito nacional, o internacional en ausencia de los primeros" y el Art. 64 del Reglamento de Seguridad para la Construcción y Obras Públicas (R0 249: 10-enero-2008) menciona en el literal 4 la Obligación de evaluar ergonómicamente el levantamiento de cargas según el método internacionalmente reconocido. ${ }^{9}$

En el presente estudio utilizamos el cuestionario Nórdico de Kuorinka ${ }^{10}$, instrumento que detecta tempranamente la existencia de síntomas músculo-esqueléticos iniciales, que todavía no han constituido enfermedad, proporcionando información que permite 
estimar el nivel de riesgos de manera proactiva y nos permite una actuación precoz. Las preguntas del cuestionario son de elección múltiple y pueden ser aplicadas en una de dos formas. Una es en forma auto-administrada, es decir, es contestado por la propia persona encuestada por sí sola, sin la presencia del investigador. La otra forma es ser aplicado directamente por el investigador, como parte de una entrevista.

Los trabajadores de mantenimiento al realizar tareas con levantamientos frecuentes, trasladar objetos livianos o pesados, manejo manual de materiales y al efectuar prolongados turnos de trabajo, pueden estar expuestos a factores de riesgos ergonómicos, que añadidos a otros factores tales como los organizacionales, individuales y ambientales, pueden ocasionar síntomas músculo-esqueléticos, afectando el estado de salud del trabajador.

Con los resultados obtenidos, se puede coordinar acciones con otras instituciones afines, y coadyuvar esfuerzos tendientes a orientar y fortalecer las normativas vigentes, y las intervenciones administrativas, en beneficio del rendimiento, la productividad y la salud ocupacional del trabajador de la empresa.

$\mathrm{Al}$ encasillarse las lesiones músculo-esqueléticas como enfermedades ocupacionales prevenibles, el propósito principal de este estudio fue determinar la prevalencia de sintomas músculoesqueléticos, en los trabajadores del área de mantenimiento de la empresa petrolera, que laboran en la zona oriental de Ecuador.

\section{METOdOLOGÍA}

Se realizó un estudio transversal en un universo poblacional de 102 trabajadores operativos, correspondientes al puesto de trabajo de mantenimiento de la empresa petrolera, en una locación ubicada en la Provincia de Sucumbíos, durante el año 2013. Los criterios de inclusión fueron los siguientes: trabajadores que de acuerdo a su puesto de trabajo, manejan herramientas y equipos para su labor en el área de mantenimiento, con un tiempo mínimo de seis meses, que sean mayores de 18 años y que llenaron el consentimiento informado. De exclusión: trabajadores de permanencia temporal menor a seis meses en el lugar de trabajo, que solamente estén reemplazando al personal considerado fijo en su puesto de trabajo, que presenten trastornos músculo-esqueléticos anteriores a la contratación y que presenten patologías congénitas. De eliminación: trabajadores que no respondan el cuestionario nórdico o quienes una vez iniciado el estudio, decidan no seguir colaborando.

En esta investigación se seleccionó a los trabajadores operativos de mantenimiento, como muestra de estudio por el tipo de actividad que realizan al emplear para sus labores, equipos y herramientas pesadas y dificiles de manipular como válvulas de bloqueo, llaves, mangueras, bombas hidraúlicas y diversos accesorios que pueden constituir niveles de criticidad, donde los trabajadores laboran en forma activa, requiriendo una demanda física intensa y en jornadas de 12 horas diarias, lo que puede desencadenar riesgo de producir sintomas músculo- esqueléticos. El estudio se llevó a cabo en la empresa petrolera, porque en ella no se han realizado investigaciones referentes al tema, y los resultados obtenidos impactarán en beneficio del cuidado de la salud de los trabajadores y la prevención de riesgos ergonómicos, para disponer de herramientas necesarias en la elaboración de procedimientos adecuados de un trabajo seguro, y contribuir a la disminución de riesgos de adquirir enfermedades músculo-esqueléticas.

Para la recolección de datos, a cada uno de los trabajadores en los puestos de trabajo de mantenimiento, se aplicó previo consenti- miento informado, los siguientes instrumentos: 1) Cuestionario de Datos Socio-Demográficos y Registros Antropométricos como peso, talla, índice de masa corporal, edad, estado civil, escolaridad, práctica de algún deporte en el trabajo, práctica de algún deporte en el descanso, trabaja en sus días de descanso, antigüedad en la empresa, antigüedad en el puesto. 2) Historia Médica Ocupacional, que fue revisada en el servicio médico de la empresa, de cada uno de los trabajadores que participaron en el estudio. La revisión incluyó especialmente los antecedentes patológicos personales como artritis, gota, diabetes mellitus; antecedentes patológicos familiares, antecedentes de lesiones músculo-esqueléticos ocurridos hace 12 meses y en los últimos 7 días, antecedentes de hospitalización, por enfermedades y/o síntomas músculo-esqueléticos y consultas de los trabajadores a los servicios médicos de la empresa por lesiones músculo-esqueléticas, en los últimos 12 meses y en los últimos 7 días. 3) Aplicación del Cuestionario Nórdico Estandarizado para detectar síntomas músculoesqueléticos en nueve regiones corporales; cuello, los hombros, la parte superior espalda, los codos, la zona lumbar, muñeca / manos, caderas / muslos, las rodillas y tobillos / pies. Kourinka et al, versión traducida en Español. ${ }^{10}$

El Cuestionario Nórdico fue desarrollado a partir de un proyecto financiado por el Consejo Nórdico de Ministros. ${ }^{10}$ El objetivo fue desarrollar y probar un sistema normalizado, que permitiera crear una metodología mediante un cuestionario de comparación de sintomas en las regiones corporales. La herramienta no fue desarrollada para el diagnóstico clínico de enfermedad osteo-muscular.

La metodología para recoger la información se realiza a través de preguntas que son de elección múltiple. En la primera sección se aplica un cuestionario general de preguntas (40) que identifican las áreas del cuerpo que causan molestias músculo-esqueléticas, en esta sección se incluye un mapa del cuerpo, que indica los nueve lugares que identifica los síntomas: en el cuello, los hombros, la parte superior espalda, los codos, la zona lumbar, muñeca / manos, caderas / muslos, las rodillas y tobillos / pies. Se pregunta a los trabajadores si han tenido sintomas músculo-esqueléticos en los últimos 12 meses y en los últimos 7 días que hayan impedido realizar su actividad normal.

En la segunda sección se describen preguntas (25) adicionales, relacionadas con hospitalización o si cambia de trabajo por presentar molestias músculo-esqueléticas, reduce actividad laboral, disminuye la actividad recreativa y acude a un profesional por presentar síntomas músculo-esqueléticas en los últimos 12 meses, y ha tenido molestías en espalda baja, espalda alta, cuello y hombros en los últimos 7 días.

Como fortaleza de medición del instrumento se menciona la amplia gama en salud ocupacional que tiene para evaluar sintomas músculo-esqueléticos, que todavía no han constituido enfermedad desde trabajadores del campo informático, del puesto de trabajo conductores ${ }^{11}$, hasta trabajadores forestales ${ }^{12}$, proporcionando información que permite estimar el nivel de riesgos de manera temprana con una actuación rápida. Su limitante radica que las distintas partes no están claramente definidas.

La fiabilidad de cuestionario nórdico se realizó utilizando una metodología de test-retest, encontrando que el número de diferentes respuestas varió entre 0 a 23\%. La Validez del instrumento encontró una congruencia con la historia clínica, comparando el dolor en los últimos 7 días y el examen clínico dando una sensibilidad que oscilaba entre el 66 y el 92\% y una especificidad entre el 71 y $88 \% .{ }^{13}$ Sin embargo, el examen médico es esencial para establecer un diagnóstico clínico. 
Todos los procedimientos de la investigación se realizaron de acuerdo con los principios bioéticos establecidos en la Declaración de Helsinki. Para evaluar el índice de masa corporal se utilizó la escala de rangos de la $\mathrm{OMS}^{14}$, los trabajadores se ubicaron en 2 categorias: no obesos aquellos trabajadores con un IMC igual o menor de $25 \mathrm{Kg} / \mathrm{m} 2$ y sobrepeso/obesidad con un IMC igual o mayor a $25 \mathrm{Kg} / \mathrm{m} 2$.

Los resultados se mostraron en valores absolutos y relativos, para la prueba de asociación se aplicó $\mathrm{chi}^{2}$ de independencia, el cual se consideró como significativo con valor de p menor o igual a 0,05. De las variables categóricas se obtuvo el valor de la distribución de las frecuencias, tablas; y en las variables socio-demográficas se calculó la desviación estándar y la media, de acuerdo a las características de los datos fueron procesados y analizados en el programa estadístico SPSS versión 20. Además se realizó una regresión logística binaria para determinar las variables que mejor explicarian la aparición de sintomas músculo-esqueléticos.

\section{RESULTADOS}

\section{Características Socio Demográficas}

La edad media de los trabajadores fue de 34,12 años (rango de 18 - 49 años), la antigüedad en la empresa fue mayor que la antigüedad en el puesto 39, 46 meses, la jornada de trabajo fue de 84 horas a la semana.

En relación a la escolaridad la mayoría de trabajadores, señaló haber realizado bachillerato, seguido del nivel técnico, no existe analfabetismo en el grupo de estudio.

Con respecto a la variable que explora si realizan algún deporte en el trabajo en sus horas de descanso, el 35\% señaló algún tipo de sintoma. Referente a la pregunta: ¿realizan deporte en sus días de descanso?, el 32\% presenta síntomas músculo-esqueléticos. Al aplicar la prueba Chi cuadrado se obtuvo diferencia significativa entre las variables antigüedad en la empresa y la presencia de síntoma músculo-esquelético en el cuello ( $\mathrm{X} 2=77,2 \mathrm{p}<0,00)$; antigüedad en la empresa con dolor a nivel de espalda baja (X2=71,43 $\mathrm{p}<0,00$ ). Asímismo se encontró asociación significativa entre la variable deporte en el trabajo y síntoma músculo-esquelético en el cuello (X2=9,92 p<0,05); y entre deporte en el trabajo con síntoma músculo esquelético en la espalda alta (X2=9,75 p<0,00).

En la variable, ¿trabajan en sus días de descanso?, más de la mitad de los trabajadores refirió laborar mientras están en sus dias de descanso, lo cual puede explicar en cierto sentido que estos trabajadores ingresen desde su hogar a laborar en la empresa, con algún tipo de molestia músculo-esquelética. Se encontró asociación significativa entre la variable trabaja en el descanso y sintoma músculo-esquelético en la espalda baja (X2=14,17 p<0,00).

Respecto al Índice de Masa Corporal, se evidenció que la mayoría de los trabajadores presenta valores superiores a $25 \mathrm{Kg} / \mathrm{m} 2$, es decir tienen sobrepeso y obesidad; 37 (36,3\%) tienen peso normal y $3(2,9 \%)$ tienen infrapeso. No se encontró asociación significativa entre el índice de masa corporal y la presencia de síntomas músculo-esqueléticos.

\section{Historia Médica Ocupacional}

En relación a los antecedentes patológicos personales, entre los trabajadores que respondieron tener un antecedente de alguna enfermedad personal, sin especificar qué tipo de patología adolecen, de ellos solamente el 8\% mencionó tener molestias músculo-esqueléticas.
En antecedentes patológicos familiares, los trabajadores expresaron tener antecedentes de enfermedades en algún miembro de su familia, especialmente hipertensión arterial, diabetes mellitus, enfermedad coronaria, aunque no especifican en forma clara qué tipo de patología adolecen sus familiares.

Respecto a antecedentes de lesiones músculo-esqueléticas, los trabajadores, manifestaron haber tenido patología músculo-esquelética pero de origen traumático (caídas, tropezones) adquiridas principalmente en actividades deportivas; solo 1 trabajador expresó haber presentado una lesión de origen ocupacional, la que fue descartada en el servicio médico de la empresa.

En cuanto a los antecedentes de hospitalización, los trabajadores que manifestaron haber estado hospitalizado en instituciones de salud públicas o privadas, a consecuencia de lesiones músculoesqueléticas, indicaron mostrar una recuperación satisfactoria. La mayoría de los trabajadores refirió acudir a un servicio de salud, a consultar con un profesional médico, por presentar alguna molestia músculo-esquelética. No se encontró asociación significativa entre las variables de la historia médica ocupacional y sintomas músculo-esqueléticos.

En la Tabla 1, se muestran las características socio-demográficas -antropométricas e historia médica-ocupacional de trabajadores operativos del puesto de trabajo mantenimiento de la empresa petrolera investigada, en donde se evidencia que un elevado número de trabajadores realizan deporte mientras están en sus días de descanso, y un porcentaje alto de trabajadores acuden a consultas médicas por presentar síntomas músculo-esqueléticos. Los trabajadores en el área de mantenimiento ocupan los siguientes

\section{Tabla 1.}

Caracteristicas Socio-Demográficas - Antropométricas e Historia MédicaOcupacional de trabajadores operativos del puesto de trabajo mantenimiento de una empresa petrolera.

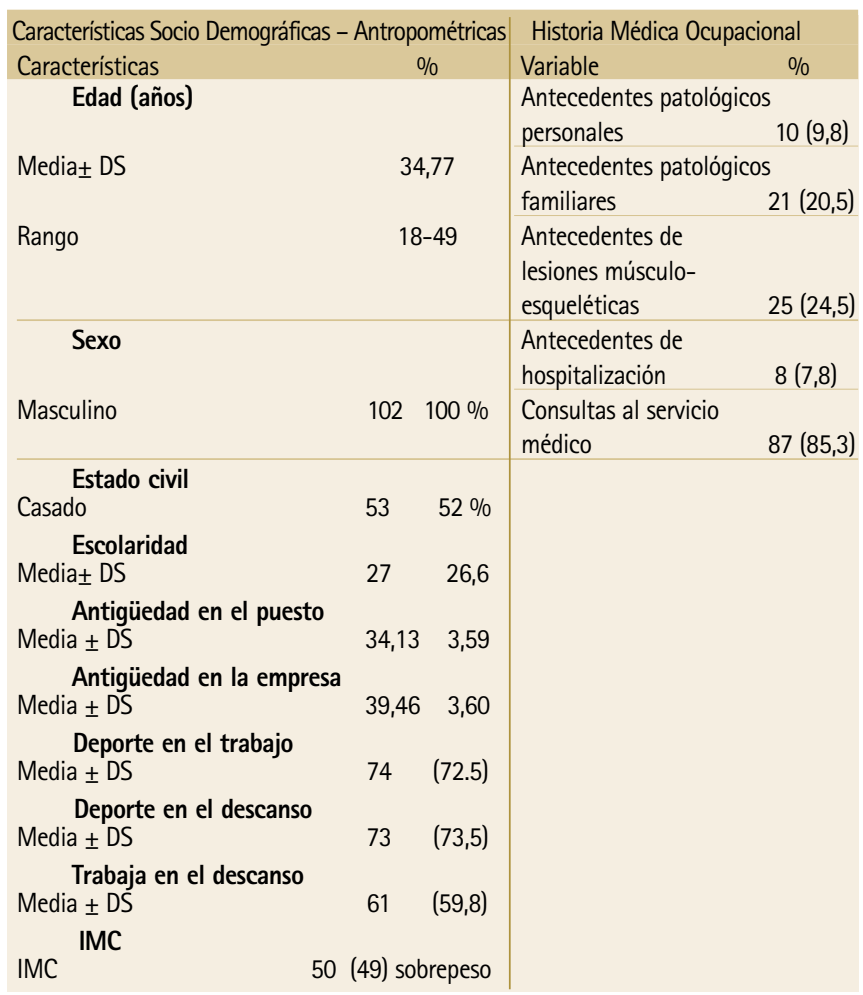

Expediente laboral y Cuestionario directo.. 
puestos de trabajo: técnico eléctrico, técnico mecánico, técnico de mantenimiento predictivo, técnico de instrumentación, técnico de integridad mecánica, técnico de automatización, técnico automotriz, ayudante mecánico, ayudante eléctrico, ayudante automotriz, bodeguero de mantenimiento, ayudante de mantenimiento predictivo, ayudante de integridad mecánica, ayudante de instrumentación, técnico de aire acondicionado y servicio de rotulación y pintura. Al aplicar la prueba de Chi cuadrado se obtuvo una diferencia significativa entre las variables puesto de trabajo y la presencia de síntoma músculo esquelético en cuello (X2=50,11 $\mathrm{p}<0,00$ ); entre puesto de trabajo y síntoma músculo-esquelético en hombro (X2=65,81 p<0,00).

En la Tabla 2, se muestra la distribución de la prevalencia de síntomas músculo-esqueléticos según los puestos de trabajo, el puesto técnico eléctrico revela la mayor prevalencia, seguido del puesto técnico mecánico.

La Tabla 3 evidencia la prevalencia de síntomas músculo-esqueléticos de acuerdo a las regiones corporales; los porcentajes más altos se obtuvieron en la espalda baja, constituyéndose en la parte del cuerpo más afectada.

La Tabla 4 evidencia la prevalencia de sintomas músculo-esqueléticos, que presentan los trabajadores operativos de mantenimiento, en los últimos doce meses en las principales zonas corporales, región dorso lumbar, cuello y hombros.

Tabla 2.

Distribución de la prevalencia de síntomas músculo-esqueléticos según el puesto de trabajo.

\begin{tabular}{|c|c|c|c|c|c|c|}
\hline \multirow[b]{3}{*}{ Puesto de trabajo } & \multirow[b]{3}{*}{$\mathrm{n}$} & \multirow[b]{3}{*}{$\%$} & \multicolumn{4}{|c|}{ Presencia de síntoma } \\
\hline & & & & & & \\
\hline & & & $n$ & $\%$ & No & $\%$ \\
\hline Técnico mecánico & 20 & $19,6 \%$ & 17 & $16,7 \%$ & 3 & $2,9 \%$ \\
\hline Técnico eléctrico & 30 & $29,4 \%$ & 23 & $22,5 \%$ & 7 & $6,9 \%$ \\
\hline Técnico de instrumentación & 15 & $14,7 \%$ & 14 & $13,7 \%$ & 1 & $1,0 \%$ \\
\hline Técnico de automatización & 2 & $2,0 \%$ & 2 & $2,0 \%$ & 0 & $0,0 \%$ \\
\hline Técnico de integridad mecánica & 0 & $0,0 \%$ & 0 & $0,0 \%$ & 0 & $0,0 \%$ \\
\hline Técnico de mantenimiento predictivo & 5 & $4,9 \%$ & 5 & $4,9 \%$ & 0 & $0,0 \%$ \\
\hline Técnico automotriz & 5 & $4,9 \%$ & 5 & $4,9 \%$ & 0 & $0,0 \%$ \\
\hline Ayudante automotriz & 1 & $1,0 \%$ & 1 & $1,0 \%$ & 0 & $0,0 \%$ \\
\hline Ayudante mecánico & 10 & $9,8 \%$ & 9 & $8,8 \%$ & 1 & $1,0 \%$ \\
\hline Ayudante eléctrico & 3 & $2,9 \%$ & 3 & $2,9 \%$ & 0 & $0,0 \%$ \\
\hline Ayudante instrumentación & 0 & $0,0 \%$ & 0 & $0,0 \%$ & 0 & $0,0 \%$ \\
\hline Ayudante de integridad mecánica & 0 & $0,0 \%$ & 0 & $0,0 \%$ & 0 & $0,0 \%$ \\
\hline Bodeguero mantenimiento & 3 & $2,9 \%$ & 3 & $2,9 \%$ & 0 & $0,0 \%$ \\
\hline Ayudante mantenimiento predictivo & 4 & $3,9 \%$ & 4 & $3,9 \%$ & 0 & $0,0 \%$ \\
\hline Técnico de aire acondicionado & 3 & $2,9 \%$ & 3 & $2,9 \%$ & 0 & $0,0 \%$ \\
\hline Servicio de rotulación y pintura & 1 & $1,0 \%$ & 1 & $1,0 \%$ & 0 & $0,0 \%$ \\
\hline Total & 102 & 1 & 90 & $88,2 \%$ & 12 & $11,8 \%$ \\
\hline
\end{tabular}

Con relación al tiempo en el que estas molestias le han impedido hacer su trabajo en los últimos 12 meses, las zonas que reportan problemas que han impedido realizar su trabajo por más tiempo son el cuello, espalda alta, y espalda baja.

Los resultados indican que las zonas donde con más frecuencia los trabajadores acuden donde un profesional en los últimos 12 meses, son en hombro (93\%), cuello (92,2\%), y espalda baja (85,3\%).

Los resultados del análisis, ¿han tenido molestias en los últimos 7 dias?, nos muestra que las zonas donde se evidenció una mayor prevalencia son en cuello, seguido de espalda baja.

En la Tabla 5 se observa la prevalencia de síntomas músculoesqueléticos, que los trabajadores presentan en los últimos 7 días. En la Tabla 6 se presenta el modelo logístico conjunto de las variables de la ecuación: socio-demográficas-antropométricas, historia médica ocupacional (antecedentes patológicos personales), en el mismo se observa que las variables no son estadísticamente significativas; es decir por sí solas no explican la aparición de sintomas músculo-esqueléticas.

Además, quienes realizan deporte en el descanso, tienen mayor probabilidad de que aparezcan síntomas músculo esqueléticos.

En la Tabla 7 se presenta la regresión logística de la variable sociodemográfica-antropométricas, se observa que el modelo no es significativo (sig. $=0,173>0,05$ ), estas variables explican un $28,2 \%$ de la aparición de síntomas músculo-esqueléticas.

Tabla 3.

Prevalencia de molestias músculo-esqueléticas - según regiones corporales.

$\begin{array}{lcc}\text { Variable } & \text { SI } & \text { NO } \\ \text { Espalda alta } & 44(43,1) & 58(56,9) \\ \text { Espalda baja } & 66(64,7) & 36(35,3) \\ \text { Cuello } & 38(37,3) & 64(62,7) \\ \text { Hombros } & 27(26,5) & 75(73,5) \\ \text { Codos } & 9(8,5) & 87(85,3) \\ \text { Manos y muñecas } & 22(21,6) & 74(72,5) \\ \text { Una 0 ambas caderas o muslos } & 16(15,7) & 77(75,5) \\ \text { Una 0 ambas rodillas } & 24(24,5) & 69(67,6) \\ \text { Uno 0 ambos tobillos o pies } & 21(20,6) & 69(67,6)\end{array}$

Tabla 5.

Prevalencia de síntomas músculo-esqueléticos en los últimos 7 días.

\begin{tabular}{lcc} 
Variable & $\begin{array}{c}\text { SI } \\
\text { Frecuencia (\%) }\end{array}$ & $\begin{array}{c}\text { NO } \\
\text { Frecuencia (\%) }\end{array}$ \\
\hline Espalda alta & $64(62,7)$ & $38(37,3)$ \\
Espalda baja & $85(83,3)$ & $17(16,7)$ \\
Cuello & $88(86,3)$ & $14(13,7)$ \\
Hombros & $10(9,8)$ & $92(90,2)$
\end{tabular}

Tabla 4.

Prevalencia de las molestias músculo-esqueléticas de los últimos doce meses en las principales zonas corporales.

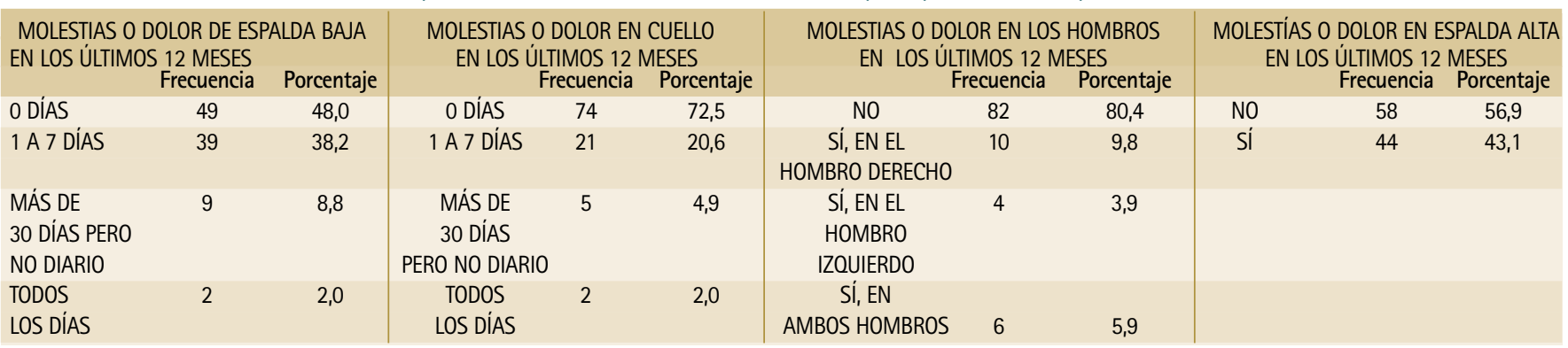


Tabla 6.

Modelo logístico conjunto-Variables en la ecuación.

\begin{tabular}{|c|c|c|c|c|c|c|c|}
\hline & & B & $\begin{array}{l}\text { Error } \\
\text { estándar }\end{array}$ & Wald & gl & Sig. & $\operatorname{Exp}(B)$ \\
\hline \multirow{11}{*}{$\begin{array}{l}\text { Paso } \\
1 \text { la }\end{array}$} & EDAD & $-0,968$ & 611,5 & 0 & 1 & 1 & 0,38 \\
\hline & Estado civil & 23,82 & 20465 & 0 & 1 & 1 & $2,2 \mathrm{E}+10$ \\
\hline & Escolaridad & 187,5 & $1 \mathrm{E}+05$ & 0 & 1 & 1 & $2,70 E+81$ \\
\hline & $\begin{array}{l}\text { Antigüedad en } \\
\text { la empresa }\end{array}$ & 0,203 & 283,1 & 0 & 1 & 1 & 1,226 \\
\hline & $\begin{array}{l}\text { Antigüedad en } \\
\text { el puesto }\end{array}$ & 0,355 & 245,1 & 0 & 1 & 1 & 1,426 \\
\hline & $\begin{array}{l}\text { Practica algún } \\
\text { deporte en } \\
\text { el Trabajo }\end{array}$ & $-32,17$ & 42766 & 0 & 1 & 1 & 0 \\
\hline & $\begin{array}{l}\text { Practica algún } \\
\text { deporte en } \\
\text { el descanso }\end{array}$ & 135,7 & 13321 & 0 & 1 & 0,99 & $8,79 E+58$ \\
\hline & $\begin{array}{l}\text { Trabaja en sus } \\
\text { dias de descanso }\end{array}$ & $-28,49$ & 9915 & 0 & 1 & 1 & 0 \\
\hline & $\begin{array}{l}\text { Antecedentes } \\
\text { patológicos } \\
\text { personales }\end{array}$ & $-61,22$ & 23229 & 0 & 1 &, 998 & 0 \\
\hline & Constante & 362,9 & 76839 & 0 & 1 & 1 & $4,01 E+157$ \\
\hline & Constante & 362,9 & 76839 & 0 & 1 & 1 & $4,01 E+157$ \\
\hline
\end{tabular}

En la Tabla 8 se evidencia la regresión logística de la variable historia médica ocupacional (antecedentes patológicos personales), donde estas variables explican un 4,7\% la aparición de síntomas músculo-esqueléticos, por lo tanto no es estadísticamente significativa.

\section{DISCUSIÓN}

Este estudio se realizó para analizar la prevalencia de los síntomas músculo-esqueléticos en trabajadores operativos de mantenimiento de la empresa petrolera; en este sentido, el porcentaje de trabajadores que respondieron que habian tenido en alguna ocasión molestias músculo-esqueléticas fue de 88,2 \%. Esta es una prevalencia muy alta, pero similar y superior a los datos de la VI Encuesta Nacional de las Condiciones de Trabajo, donde el 74,2\% de los trabajadores encuestados manifestaron sentir alguna molestia músculo-esquelética. ${ }^{15}$

Tabla 7.

Regresión Logística Variable Socio Demográfica-Antropométrica.

\begin{tabular}{|c|c|c|c|c|c|c|c|c|c|}
\hline $\begin{array}{l}\text { Pruebas ómnibus } \\
\text { de coeficientes } \\
\text { del modelo }\end{array}$ & & $\begin{array}{c}\text { Chi- } \\
\text { cuadrado }\end{array}$ & gl & Sig & $\begin{array}{l}\text { Resumen del } \\
\text { modelo } \\
\text { logístico }\end{array}$ & Escalón & $\begin{array}{l}\text { Logaritmo de } \\
\text { la verosimilitud } \\
-2\end{array}$ & $\begin{array}{c}\mathrm{R} \\
\text { cuadrado de } \\
\text { Cox y Snell }\end{array}$ & $\begin{array}{l}\text { R cuadrado } \\
\text { de } \\
\text { Nagelkerke }\end{array}$ \\
\hline $\begin{aligned} \text { Paso } 1\end{aligned}$ & Escalón & 33,75 & 27 & 0,173 & & 1 &, $000 a$ & 0,282 & 1 \\
\hline & Bloque & 33,75 & 27 & 0,173 & & & & & \\
\hline & Modelo & 33,75 & 27 & 0,173 & & & & & \\
\hline
\end{tabular}

Tabla 8.

Regresión Logística Variable Historia Médica Ocupacional.

\begin{tabular}{|c|c|c|c|c|c|c|c|c|c|}
\hline $\begin{array}{l}\text { Pruebas ómnibus } \\
\text { de coeficientes } \\
\text { del modelo }\end{array}$ & & $\begin{array}{c}\text { Chi- } \\
\text { cuadrado }\end{array}$ & gl & Sig & $\begin{array}{l}\text { Resumen del } \\
\text { modelo } \\
\text { logístico }\end{array}$ & Escalón & $\begin{array}{l}\text { Logaritmo de } \\
\text { la verosimilitud } \\
-2\end{array}$ & $\begin{array}{l}\text { R } \\
\text { cuadrado de } \\
\text { Cox y Snell }\end{array}$ & $\begin{array}{l}\text { R cuadrado } \\
\text { de } \\
\text { Nagelkerke }\end{array}$ \\
\hline Paso 1 & Escalón & 4,864 & 8 & 0,772 & & 1 & $28,887 a$ & 0,047 & 0,165 \\
\hline & Bloque & 4,864 & 8 & 0,772 & & & & & \\
\hline & Modelo & 4,864 & 8 & 0,772 & & & & & \\
\hline
\end{tabular}

La mayor prevalencia de síntomas músculo-esqueléticos, se observó en el nivel de escolaridad técnico, probablemente debido a que estos trabajadores, quienes tienen escasa capacitación y limitante nivel de instrucción, deciden laborar en trabajos con mayores factores de riesgo laborales especialmente físico y ergonómico. Contrariamente en trabajadores de una empresa de cemento Robaina, León y Sevilla ${ }^{16}$ manifestaron que el grupo con mayor número de casos de síntomas músculo-esqueléticos, correspondía a trabajadores que disponian de estudios de secundaria y preuniversitarios.

El análisis de los resultados, reveló que el 53\% de los trabajadores sufrió trastornos músculo-esqueléticos en la espalda baja y alta (zona lumbar y dorsal) en los últimos 12 meses, resultado muy parecido al de Garcia, Switt y Ledesma ${ }^{17}$, cuyo porcentaje fue del 60\%. Estos resultados están en relación al estudio de Bingefors e Isacson ${ }^{18}$, donde manifiestan que el dolor de espalda es uno de los problemas médicos más comunes de la población en general. sintomas músculo-esqueléticos y la edad; el porcentaje mayor de casos se evidenció en trabajadores jóvenes, 30-40 años, grupo donde se encuentra la mayor población de trabajadores de la empresa petrolera. A partir de los 41 años se observa un ligero descenso de los síntomas; lo que explicaría que los trabajadores a través de los años aplican las medidas necesarias para disminuir y controlar los riesgos, además de la antigüedad en la empresa y las promociones laborales que por sus habilidades y desempeño los colocan en otros puestos, donde realizan menor carga laboral y por ende tienen menor riesgo de contraer este tipo de lesiones.

Los hallazgos encontrados en el estudio coinciden con lo reportado por Merlino et al. ${ }^{2}$, donde no existió asociación entre el índice de masa corporal y los síntomas músculo-esqueléticos.

Los resultados de este estudio coinciden con otras investigaciones donde en poblaciones jóvenes fue encontrado el mayor número de casos, en los cuales no hubo asociación entre la edad y la presencia de síntomas músculo-esqueléticos, probablemente por las distintas condiciones, exigencias y duración de las actividades ocupacionales efectuadas por los trabajadores estudiados. ${ }^{16,19,20}$

En este estudio las regiones anatómicas más frecuentemente afectadas fueron espalda alta, espalda baja, cuello y hombro, lo que concuerda con la investigación realizada por Goldsheyder et al. ${ }^{21}$ ciones ergonómicas del trabajo Page-Garcia-Molina et al. ${ }^{22}$, contrastados con datos personales de los trabajadores, sugieren que en la aparición de estos síntomas, también intervienen diversos factores como el nivel de práctica de actividad física, el hábito tabáquico, el
En este estudio no se encontró relación significativa entre los

La asociación entre los síntomas músculo-esqueléticos y las condi- 
género, factores psicosociales, genéticos, etc, descritos en diversos estudios. ${ }^{23-25}$

Considerando que existe un elevado porcentaje de trabajadores operativos del área de mantenimiento que padece síntomas músculoesqueléticos, será necesario efectuar acciones tendientes a prevenir la aparición de estos sintomas. Gómez y Valero ${ }^{26}$ sugieren que los trabajadores realicen en las organizaciones, especialmente en las horas de descanso, ejercicios físicos de estiramientos y técnicas de relajación.

Holtermann, Blangsted, Christensen, Hansen, Soogard y Hyoung ${ }^{27,28}$ recomiendan dar mayor importancia a la fuerza muscular para conservar una adecuada salud músculo-esquelética y evitar este tipo de lesiones.

\section{CONCLUSIONES}

Los trabajadores objeto de estudio revelaron una elevada prevalencia de sintomas músculo-esqueléticos, especialmente en espalda baja, espalda alta, cuello y hombro, debido a que, presuntivamente, en las actividades que ellos realizan efectúan levantamiento de cargas, manipulación manual de materiales y herramientas, que pueden aumentar el riesgo de aparición de lesiones músculo-esqueléticas.
Al aplicar la prueba Chi cuadrado se obtuvo diferencia significativa entre las variables antigüedad en la empresa y la presencia de síntoma músculo- esquelético en el cuello y antigüedad en la empresa con dolor a nivel de espalda baja. Asímismo se encontró asociación significativa entre la variable deporte en el trabajo y síntoma músculo-esquelético en el cuello y asociación entre la variable trabaja en el descanso y síntoma músculo-esquelético en la espalda.

Las labores en las empresas petroleras, representan una de las áreas más riesgosas de la industria en el desarrollo de los sintomas músculo-esqueléticos, se recomienda efectuar una intervención ergonómica y de capacitación a los trabajadores de la empresa, para reducir la frecuencia de los síntomas mediante la adopción de medidas técnicas, administrativas y organizacionales de la empresa donde laboran.

De acuerdo al análisis de los puestos de trabajo, el puesto técnico eléctrico presenta la mayor prevalencia de síntomas músculo-esqueléticos, seguido del puesto de trabajo técnico mecánico, por lo cual es necesario una intervención ergonómica en la empresa.

$\mathrm{Al}$ desarrollar la regresión logística con las tres variables del estudio, se concluye también que el modelo logístico de las variables investigadas no es estadísticamente significativo. 
1. Kumar S. Theories of muskuloskeletal injury causation. Ergonomics. 2001; 44(1):17-47.

2. Merlino, L, Rosecranse, J, Antón, D y Cook, T. Symptoms of musculoskeletal disorders among apprentice constructión workers. Appl Occup Environ [en línea] 2003 [citado 10 oct 2013] 18(1):57-64. Disponible en: www.scielo.org. ve/scieloOrg/php/articleXML.php?pid

3. Verdú NR, Villanueva MA. Seguimientos de daños para la salud por transtornos músculo-esqueléticos. Instituto de seguridad y salud laboral. [Póster]. 1er Congreso de Prevención de Riesgos Laborales; 1 y 2 de marzo 2007; Murcia, España. Murcia: Instituto de Seguridad y Salud Laboral; 2007.

4. Organización Internacional del Trabajo. La salud y la seguridad en el trabajo y Ergonomia [en línea] 2005 [citado 20 dic 2012]. Roma: OIT; [s.f.]. Disponible en: http://training.itcilo.it/actrav_cdrom2/es/osh/ergo/ergonomi. htm.

5. Pruss $\mathrm{A}$, Convalán $\mathrm{CF}$, Pastides $\mathrm{H}$, Hollander $\mathrm{A}$. Methodologic considerations in estimating burden of disease from environmental risk factors at national and global levels. International J Occup Environ Health; 2001; 7(1):58-67.

6. España. Ministerio de Empleo y Seguridad Social. Anuario de Estadisticas [en línea] 2001 [citado 8 sep 2013]. Madrid: MESS; 2002. Disponible en: http:// www.mtin. es/estadisticas/anuario2001/welcome.htm.

7. Troconis F, Palma A, Montiel M, Quevedo A, Rojas L, Chacin B, et al. Valoración postural y riesgo de lesión músculo-esquelética en trabajadores de una plataforma de perforación petrolera lacustre. Salud de los Trabajadores. 2008; 16(1):29-38.

8. Costo enfermedad ocupacional. Entrevista a Juan Vélez. El Mercurio, Cuenca, Ecuador [en línea]. 28 Abr 2013 [citado 10 oct 2013]. Disponible en: http:// www.elmercurio.com.ec/378569-siniestralidad-laboral-es-alta-en-el-ecuador/

9. Carrera GD. Análisis de los riesgos ergonómicos y psicosociales en trabajadores expuestos a turnicidad, pdv, carga fisica, mental, desarraigo familiar en una empresa petrolera ubicada en la Provincia de Orellana Ecuador [tesis]. Quito: Universidad Internacional SEK; 2012.

10. Kuorinka I, Jonsson B, Kilbom A., Vinterberg $H_{\text {., }}$ Biering-Sorensen $F_{\text {, }}$ Andersson G, Jorgensen K. Standardised Nordic questionnaires for the analysis of musculoskeletal symptoms. Appl Ergon. 1987; 18(3):233-237.

11. Porter JM, Gyi DE. The prevalence of musculoskeletal troubles among car drivers. Occup Med. 2002; 52(3):4-12.

12. Hagen $K$, Magnus $P$, Vetlesen $K$. Neck/shoulder and lowback disorders in the forestry industry: relationship to work tasks and perceived psychosocial job stress. Ergonomics. 1998; (41):1510-1518.

13. Ohlsson K, Attewell $R$, Johnsson B. An assessment of neck and upper extremity disorders by questionnaire and clinical examination. Ergonomics. 2000; 37(2):891-897.

14. Organización Mundial de la Salud. Estrategia mundial sobre régimen alimentario, actividad física y salud. Ginebra: OMS; 2004.

15. Medical Physics [en línea]. Trastornos músculo- esqueléticos como primera causa de baja en relación con las condiciones de trabajo, según VI Encuesta Nacional de las Condiciones de Trabajo de España, Instituto de Seguridad e Higiene en el Trabajo. Santa Cruz de Tenerife: Medical Physics; 2007 [citado nov 2013]. Disponible en: www.medicalphysis.com/documents/prevencionderiesgosLaborales

16. Robaina C, León I, Sevilla D. Epidemiología de los trastornos osteomioarticulares en el ambiente laboral. Rev Cubana Med Gen Integr [en línea] 2000 [citado 2 jul 2014] 16(6):531-539.Disponible en: http://www.bvs.sld.cu/ revistas/mgi/vol16_1_00/mgi02600.htm
17. Garcia C, Switt M, Ledesma A. Trastornos musculoesqueléticos de espalda lumbar en trabajadores de la salud de la comuna rural de Til Til. Cuadernos Médico Sociales. 2007; 47(2):68-73.

18. Bingefors K, Isacson D. Epidemiology, co-morbidity, and impact on healthrelated quality of life of self-reported headache and musculoskeletal pain; a gender perspective. Eur J Pain. 2004; 8(5): 435-450.

19. Rosecrance J, Proszazas J, Cook T, Fekecs E, Merlino L, Anton D. Musculo skeletal disorders among construction apprentices in Hungary. Cent Eur J Public Health. 2001; 9(4):183-7.

20. Welchs L, Hunting K, Kellogg J. Work related Musculoskeletal symptoms among sheet metal workers. Am J Ind Med. 1995;27(6):783-91.

21. Goldsheyder D, Shecter W, Nordin M, Hierbert R. Selfreported Musculoskeletal disorders among mason tenders. Am J Ind Med. 2002; 42(5): 384-396.

22. Page A, Garcia-Molina C. Ergonomía. Carga física. En: Ruiz-Frutos C, Garcia AM, Delclòs J, Benavides FG, editores. Salud laboral: conceptos y técnicas para la prevención de riesgos laborales. Barcelona: Masson; 2007. p. 381-95.

23. Ghasemkhani $M$, Mahmudi $E_{1}$ Jabbari $H$. Musculoskeletal Symptoms in Workers. Int J Occup Saf Ergo. 2008; 14(4):455-462.

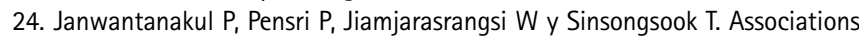
between Prevalence of Self-reported Musculoskeletal Symptoms of the Spine and Biopsychosocial Factors among Office Workers. J Occup Health. 2009; 51(2):114-122.

25. Kamaleri Y, Natvig B, Ihlebaek C, Benth J y Bruusgaard D. Number of pain sites is associated with demographic, lifestyle, and health-related factors in the general population. Eur J Pain. 2008; 12(6):742-748.

26. Gómez M, Valero A. Importancia de la actividad físico-deportiva en el mundo laboral y su repercusión en la empresa. Habilidad Motriz [en línea] 2007 [citado 25 nov 2013] 29: 47-53. Disponible en: www.educacion fisicalajarcia.blogspot.com2007_10_01_archive.html-

27. Holtermann A, Blangsted A, Christensen H, Hansen K, Soogard K. What characterizes cleaners sustaining good musculoskeletal health after years with physically heavy work? Int Arch Occup Environ Health. 2009; 82(8):1015-1022.

28. Hyoung HK. Effects of a Strengthening Program for Lower Back in Older Women with Chronic Low Back Pain. J Korean Acad Nurs. 2008; 38(6): 902-913.

\section{Referencias complementarias}

Crawford J. The Nordic musculoskeletal questionnaire. Occup Med. 2007; 57(4):300-301.

España. Ministerio de Trabajo e Inmigración. Guía técnica para la evaluación y prevención de los riesgos relativos a la manipulación manual de cargas [en linea]. Madrid: INSHT; 2003 [citado nov 2013]. Disponible en: http://www. insht.es/InshtWeb/Contenidos/Normativa/GuiasTecnicas/Ficheros/cargas.pdf

Venezuela. Instituto Nacional de Prevención, Salud y Seguridad Laborales. Registro de accidentes y enfermedades ocupacionales. Caracas: IPSSL; 2006.

Bartolomé J, Borrero J, Paramio J, Carmona J, Sierra A. Trastornos músculoesqueléticos en el personal de administración y servicios de la Universidad de Huelva. Revista Digital de Salud y Seguridad en el Trabajo. 2009; 12(1): 1-20. 